\title{
The Promotion and Effect of Ultrasound on Chinese Medicine Extraction Min Yang ${ }^{1, a}$ \\ ${ }^{1}$ Jilin Agricultural Science and Technology University, Jilin, Jilin, 132101 \\ aemail,
}

Keywords: Promotion, Effect, Ultrasound, Chinese Medicine Extraction

\begin{abstract}
Application of the principle of ultrasonic technology in traditional Chinese medicine extraction methods and the current major application for a review presentation, noted that ultrasonic extraction, their applications in medicine production, suggesting that ultrasound technology in traditional Chinese medicine extraction of the need for further the study.
\end{abstract}

\section{Introduction}

Conventional methods of traditional Chinese medicine has a boiling extraction method, water distillation, solvent extraction method, which extracts a long time, the impact of proposed rate. With the development of modern science, ultrasound technology has been widely used, but the ultrasound technique for extracting the active ingredients of traditional Chinese medicine in recent years was only seriously. Natural herbal ingredients used mostly intracellular product, when you need to extract cell disruption, and the existing mechanical or chemical methods are sometimes difficult to achieve the desired crushing effect. Studies have shown that the use of ultrasonic vibrations generated by strong, high acceleration, intense cavitation, agitation, etc., can accelerate the drug active ingredient into the solvent, thereby enhancing the proposed rate, shorten the extraction time and solvent savings, and eliminates the high temperature extraction Effect of components.

\section{Ultrasound Studies}

Ultrasonic wave propagation in the medium can enter the medium particle vibration propagation space in its state, strengthen the solute diffusion, transfer, namely ultrasonic mechanism. Ultrasonic propagation in the medium particle whose energy is constantly absorbing medium particle into heat, leading to medium particle temperature that Ultrasound thermal mechanism. At the same time when a large amount of an extraction medium to ultrasonic vibration in diluted form, the media was torn into many small holes, the small holes instantaneous closed, generating instantaneous pressure up to several atmospheres closed cavitation.

In the ultrasound field due to being broken and the like in which the extraction medium contains large amounts of dissolved gases and tiny impurities, which are surrounded by broken and the like around the outer membrane of glial for ultrasonic provide the necessary conditions. Tremendous pressure generated in the air caused by broken cell wall rupture and the whole organism, and the whole rupture process in the instantaneous vibration effect while the ultrasonic generator is enhanced release of intracellular diffusion and dissolved substances. Ultrasonic disintegration process is a physical process, the extraction process no chemical reaction, by extraction of the biologically active substance remains unchanged in a short time, not diminished the biological activity, while increasing the speed of the crusher, the crushing time was shortened, greatly improve the extraction efficiency. Also ultrasonic unifying role can not be ignored, Lin Cuiying, etc. The ultrasound has the suspension in a gas or liquid particles to agglomerate into larger particles and precipitation effects, design conventional filtered extract with an ultrasonic treatment, the rutin molecule more fast collide condense into large particles to settle to make a more complete extraction of rutin.

When the ultrasonic effect depends not only on the intensity and frequency of the ultrasonic waves, and have a certain relationship with the structure and function of the object to be crushed. Calculations show that: in the water will be produced when the ultrasonic intensity of $3000 \mathrm{~W} / \mathrm{m} 2$ 
when cavitation bubbles in the moment quickly closed, the pressure pulse generated when closed to form a spherical shock wave instantaneously, causing an organism to be broken and the cell radiating complete rupture, you can choose a suitable ultrasonic frequency is determined after theoretically be crushed in the medium in the bubble size. Because the extraction medium bubble size is not a single, but there is a distribution, so there should be a range of ultrasonic frequency change, that there is a bandwidth.

\section{The Basic Principle of Ultrasound}

Ultrasonic frequency is higher than $20 \mathrm{kHz}$, and does not cause the elastic wave hearing. Is now generally agreed that its cavitation, thermal and mechanical action of ultrasound technology in traditional Chinese medicine extraction of the three theoretical basis.

Cavitation in liquids often, there are some vacuum or gas or vapor containing a small amount of small bubbles, these small bubble size varies. When the ultrasonic wave at a certain frequency of the liquid, only the size of vesicle suitable resonance phenomenon can occur, the resonance is larger than the size of the vesicles is driven out of the liquid, the size of the vesicles than the resonance at ultrasonic gradually larger. Near the resonance size, sparse acoustic stage make vesicle rapidly swell; the acoustic compression phase, vesicles suddenly compressed insulation until annihilation. Annihilation process, internal vesicles up to high temperatures and high pressure of several thousand atmospheres of thousands of degrees. The above phenomenon is called cavitation. When vesicles swollen due to friction can generate a charge in the annihilation process can produce a discharge, luminous phenomenon. Cavitation is used for cleaning, atomizing, and promote chemical reactions aspect emulsification.

Thermal effects. Due to internal friction and ultrasonic absorption media consumption, molecular excessive vibration, ultrasonic mechanical energy into internal energy of the medium, causing the medium temperature. The greater the intensity of ultrasound, thermal effect generated stronger. Ultrasonic intensity control, drugs can instantly elevated temperature within the organization, speed up the dissolution of the active ingredient, and does not change the nature of ingredients.

Mechanical action. Ultrasound is a mechanical vibration energy propagation can be formed effectively agitated flow in the liquid, damage the structure of the medium, the pulverization of the liquid particles, to achieve the common low frequency less than the effect of mechanical agitation. Commonly used in the mechanical action of crushing, cutting, coagulation and so on.

Ultrasound can not be normal temperature and pressure so that the chemical reaction occurring in the cavitation, and even the very hard solids are pulverized. Control certain frequency and intensity of ultrasound, cavitation generated tremendous pressure caused by rupture of the biological cell wall and the whole organism, and the whole crushing process is completed in an instant, while the ultrasonic vibration generated by strengthening the role of the release of intracellular material, diffusion and dissolution . Leached material being broken instantaneously biological activity unchanged, while increasing the speed of crushing and extraction rate. Ultrasound can also activate certain enzymes and cellular processes involved in physiological chemistry by changing the reactant mass transport mechanism, increase the activity of enzymes to accelerate cell metabolism.

In addition to the three principles of action, but also found that ultrasound can make agglomerate into larger particles precipitate was suspended in a gas or liquid particles, unifying role in improving the extraction rate and shorten the extraction time play an important role. Coacervation and ultrasonic time, intensity and frequency. Sound intensity is high, can achieve good flocculation effect in less time. Ultrasound also cohesion and the particle size, the nature and concentration.

Factors Extraction of Traditional Chinese Medicine

Thermal effects of ultrasound, mechanical effect, cavitation effects are interrelated. By controlling the frequency and intensity of ultrasound can highlight one of these effects, reduce or avoid further action in order to achieve the purpose of improving the extraction rate of the active ingredient. Thermal effects of ultrasound effect on the organism generated by the ultrasonic frequency significantly affected. Generally, the lower the ultrasonic frequency, the stronger the 
cavitation generated, crushing, broken and so on. So that the strong influence of cavitation cavitation bubbles generated swift solvent instantaneous collapse, prompting the plant tissue cells rupture, solvent penetrate into the plant cells, the cells of the active ingredient into the solvent to accelerate the mutual penetration and dissolved. Therefore, under ultrasound, no heating also increase the extraction rate of active ingredient. Many experiments show that the active ingredient in traditional Chinese medicine extracted under conditions of low-frequency ultrasound, does not change the drug ingredients.

The higher the frequency of the ultrasonic more likely to obtain a larger sound intensity. Taking into account the degree of interaction with the media ultrasound, the ultrasound intensity and more decisive role. Under normal circumstances, when the ultrasound intensity $0.5 \mathrm{WPcm} 2$, has been able to generate strong cavitation. Different intensity ultrasound Ectraction powder Motherwort total alkaloids found that the extraction rate decreases with increasing intensity of the ultrasound. Extract anthraquinone from rhubarb rhubarb, with the increase of ultrasonic intensity, the extraction rate of change is not too large. But $0.5 \mathrm{WPcm} 2$ low intensity ultrasonic extraction was highest, and $5,10,50 \mathrm{WPcm} 2$ intensity ultrasonic extraction rate is less than the former. The crude amount of low-intensity ultrasound with $0.28 \mathrm{WPcm} 2$ dangshen saponin extracted was nearly twice the conventional method, the purity of the content is higher than the conventional method. Drug extraction ultrasonic intensity on the report was too small, the ultrasonic intensity on the extraction rate of the drug, especially on the nature of the active ingredient should be further studied in the future.

The biggest advantage of ultrasonic extraction method is high yield, no heating, but also greatly shorten the extraction time. Ultrasonic extraction in with rhubarb anthraquinones extraction rate $3 \mathrm{~h}$ $10 \mathrm{~min}$ extraction ratio is higher by boiling method. For baicalin, 10min ultrasonic extraction extraction rate is higher than the boiling method 3h. Sophorin to extract rutin, extracted by ultrasonic extraction 10min 50min raised higher than the thermal base. Rutin ultrasonic extraction $40 \mathrm{~min}$, rutin its yield is 1.7 to 2 times the size of the production yield, savings of 30 to $40 \%$ of the original ingredients and so on. Extraction time and the extraction rate of the drug on the active ingredients of traditional Chinese medicine has attracted widespread attention. Generally have three cases: 1 Some active ingredients extraction rate increases with increasing sonication time; ${ }^{\circ}$ ultrasonic extraction rate increases with time gradually increased, after a certain time, ultrasonic time be extended, slowly increase the extraction rate; »extract rate increases with ultrasonic time, to reach a limit at some point later, but the extraction rate decreases.

After an active ingredient in the drug caused a certain time ultrasonic extraction rate of increase or slow the downward trend may be two reasons: First, the role of ultrasound in a long time, the active ingredient degradation, resulting in the extraction rate decreased; two ultrasonic time is too long, so that impurities extracted crude increase, but lower active ingredient content, the impact of increasing the extraction rate.

For a different role system, ultrasonic parameters required for different choices. Much remains to be done in the ultrasonic parameters and conditions for selection. Ultrasound techniques for pharmaceutical active ingredients or active site of extraction, often with traditional extraction methods combined.

\section{Ultrasonic Extraction Applications}

Extraction of active ingredients from plants by conventional methods are generally time-consuming, labor intensive, low efficiency, while the use of ultrasound technology has to receive a significant effect. Demagglo and other alkali extracted from Datura stramonium leaves, extraction of high alkali content $3 \mathrm{~h}$ sample of $9 \%$ by the conventional method using ultrasonic extraction 30min boiling over. Rose and other alkaloids its root will make all the wood species from Luofu, was impregnated with the conventional method for an 8h, and using ultrasonic extraction method only 15min. Ovadia and other alkaloids extracted from ipecac extracted 30s more than the Soxhlet extraction method 5h of alkali with ultrasonic waves. Ultrasound is used to extract from berberine in berberine, berberine extraction rate $30 \mathrm{~min}$ obtained by alkaline soaking than $50 \%$ higher than 
24h.

Application of ultrasonic extraction from Rhubarb Anthraquinones show that: ultrasonic treatment $10 \mathrm{~min}$, the total extraction rate of 95.52 percent, while boiling $3 \mathrm{~h}$, the total extraction rate was 63.27\%; ultrasonic extraction 20min, the extraction rate of $99.82 *$; paper chromatography and HPLC analysis of the product was extracted two methods showed that ultrasonic treatment had no effect on the structure of the product. Guo Xiaowu et Extraction berberine from rhizome when, respectively, ultrasonic treatment time, ultrasonic frequency and concentration of sulfuric acid were studied. The results showed that with $20 \mathrm{khz}$ ultrasonic extraction and soaking $24 \mathrm{~h}$ 30min same extraction rate $(8.12 \%)$, nuclear magnetic resonance spectrometer to study the extraction of the product, indicating that ultrasound has no effect on the structure of berberine. Yang and other selected SBC-300 ultrasonic processor traditional Chinese medicine (Shandong Ji Ning Zhongguan ultrasonic limited liability company), three kinds of red medicine plaster paste sonication, compared with pharmacopoeia method, ultrasonic method when significant, the provincial solvent operation simple, general ultrasound extracted three times, each time to $20 \mathrm{~min}$. And according to pharmacopoeia dipping, shaking frequently, replace ether impregnation times, the extraction time is generally complete $48 \mathrm{~h}$, time-consuming and a large amount of solvent. Gastrodin extracted from Tianma and Tianma aglycone, ultrasonic extraction with Soxhlet extraction $2 \mathrm{~h} 6 \mathrm{~h}$ of the same content; using ultrasonic extraction 10min extraction than cold soak 48h gastrodin amount is higher.

Phycobilisome is light-harvesting pigment in some algae, the spectral properties of Phycobilisome must be complete Phycobilisome. When using chemical and mechanical methods are not broken from Gracilaria algae obtain the desired Phycobilisome, with a frequency of 20-50khz, voltage 60v ultrasonic extraction 10min to get the full Phycobilisome.

Studies have shown that the role of ultrasound can activate certain enzymes and cells involved in physiological and biochemical processes, thereby enhancing the activity of the enzyme, accelerate cell metabolism. Ultrasound is used to degrade chitin, speed, low cost, the same amino acid content; extraction of fungal polysaccharides, such as Chinese caterpillar fungus polysaccharides, lentinan, Hericium polysaccharide used, compared with the traditional process, the extraction rate, the reaction process without material loss and side effects.

\section{Conclusion}

The ultrasonic extraction of traditional Chinese medicine extraction has shown obvious advantages, ultrasonic technology have different roles in Chinese herbal ingredients extracted at different stages, this extraction method behind the long production cycle of Chinese medicine large-scale production, to provide more scientific process conditions, have application value. Although ultrasonic extraction method is currently done some research, but only on a small scale laboratory for some of the individual specific object extraction process simple experimental conditions. Thus, the ultrasonic extraction of natural ingredients for the plant, the coping mechanism of action in-depth study in order to establish a more general pattern, to provide a basis for the different extraction target operating conditions.

\section{References}

[1] Huangbao Min. Traditional Chinese Medicinal Research [J]. 1998, 11 (5): 56,581.

[2] Ji Dahong, Surui Jiang, Wang Ying. MATERIA MEDICA [J]. 2000, 11 (4): 369, 3701.

[3] Wang Changli, Yang Jingliang. Shaanxi College [J]. 1993,16 (3): 33,351.

[4] Guo Xiaowu, Lin Shuyu, Rui E. Shaanxi Normal University (Natural Science) [J]. 1996, 24 (1): $50,521$.

[5] Guo Xiaowu. Chinese Pharmaceutical Journal [J]. Modern Applications, 1999,16 (3): 18,201.

[6] Guo Xiaowu, Zhang Fucheng, Lin Shuyu. Shaanxi Normal University (Natural Science) [J]. 1995,23 (1): 44,461. 